\title{
Complexity and simplification in the development of nanomedicines
}

\author{
"...we have to identify the minimal level of complexity that still leads \\ to a therapeutic effect but also allows for clinical translation."
}

Keywords: EPR • nanomedicine $\bullet$ SMART

In the beginning of the 20th century, Paul Ehrlich envisioned site-specific therapies with his famous words: "Wir müssen lernen, magische Kugeln zu gießen, die gleichsam wie Zauberkugeln des Freischützen nur die Krankheitserreger treffen" (We have to learn how to cast magic bullets, which behave like the magic bullets of a marksman and exclusively hit pathogens). The simple idea of the magic bullet has fascinated scientists in various fields for more than a century [1]. While magic bullets have been envisioned by Paul Ehrlich as small molecules targeting mutated proteins, many scientists envision nowadays nanometer-sized devices, so called nanomedicines $[2,3]$. In general, nanomedicines are defined as the application of nanotechnology for medical purposes and, therefore, combine in vitro diagnostics (sensors and analytics), in vivo diagnostics (probes and devices) as well as drug therapeutics (delivery systems and active nanocompounds) in one term. Among those systems, in particular probes and drug delivery systems consist of multiple components, including, for example, polymers and/or lipids as carrier materials, drug molecules or markers and targeting ligands, and they aim to deliver drugs specifically to pathological sites, while preventing their accumulation in healthy organs and tissues. However, achieving such a beneficial biodistribution and exploiting it to significantly improve the balance between drug efficacy and toxicity has turned out to be more difficult than expected since Ehrlich's conceptual idea. The current state of drug delivery systems is described by Twan Lammers with the term SMART, meaning 'Systems (slightly)
Modulating Adverse Reactions and Toxicity' or 'Systems (somewhat) More Able to Reach the Target site' [4]. For the vast majority of drug delivery systems evaluated over the years, these are maybe the only two things one can confidently claim for 'smart' nanomedicine formulations.

Although the possibilities of nanomedical devices are enormous, clinical success of nanosized drug delivery systems is, however, still far behind these great expectations which are certainly influenced by the idea of a magic bullet [5]. Besides a few antibodies [6], liposomal drug formulations [7], proteinpolymer conjugates [8] or long active release systems [9] for local application, especially nanosized drug delivery systems have so far failed to successfully be translated into broad clinical application. Without any doubt, the reasons for this limited success can be attributed to the inherent complexity of the human organism and the arising demands for an efficient probe or carrier system. But maybe, the moderate success also relates back to the way research on complex systems and in particularly multidisciplinary research is often conducted.

The inherent challenge of nanomedicine is its multidisciplinary complexity. Therefore, the complex problem of treating a certain disease in a patient is dissected into a multitude of discrete problems, which can be tackled separately. This approach is based on the idea that afterwards, the individual solutions can be combined to solve the whole problem. Yet, this approach contains two inherent problems. First, the dissections are only meaningful whenever individual approaches

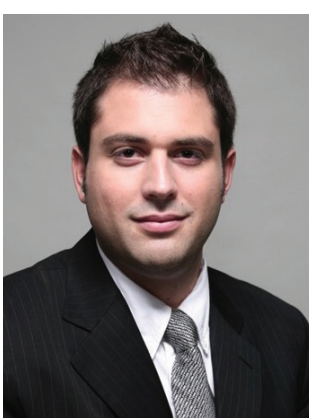

Matthias Barz

Department of Organic Chemistry, Faculty of Chemistry, Pharmaceutical Science \& Geoscience, Johannes Gutenberg-Universität Mainz, Mainz, Germany

Tel: +4961313926256

Fax: +4961313924778

barz@uni-mainz.de 
do not interfere or influence each other. Second, on top of the complex scientific task, there is the complexity arising from co-working with scientists from different disciplines. Even though it is quite clear that drug development requires multidisciplinary collaborations, a second space of complexity is generated. First of all, communicational barriers between disciplines need to be bridged, transversatility achieved, research processes aligned and discussed between scientists as well as communicated to the public. Therefore, intrinsic complexity is often reduced at all levels by simplification and findings are generalized. Without any doubt the process of simplification is necessary for communication even between scientist of different disciplines but especially in communication with the public. But what seems meaningful in the first place can easily lead to wrong assumptions when generalizations are not recognized as such and taken for granted by other researchers. This process easily leads to oversimplification and overgeneralization.

\section{"...the prejudice remains - at least in polymer science - that the polymeric material or nanoparticles need to be well-defined (narrow size or molecular weight distribution) to enable clinical translation."}

The most obvious overgeneralization starts with terms like 'nanomedicine' or 'nanoparticle'. The latter suggests that it is reasonable to group particles of a certain size independent from their chemical nature or physicochemical properties (hydrophilicity/hydrophobicity, density or charge). It is, however, very clear that a (core cross-linked) polymeric micelles has different surface or corona properties than an organic or inorganic colloid, although sizes are comparable [10]. Thus, general statements on nanoparticle properties are intrinsically prone to overgeneralization and may be the source of many problems in the development of nanomedicines.

In the following paragraphs, further cases will be described, in which oversimplification or overgeneralization compromise the development of nanomedicines.

Starting from the synthesis of nanomedicines, a first reason for the limited success of translation can be the internal complexity of the nanomedicine itself, but also the way synthesis is communicated. A demanding and skillful synthesis and characterization of multifunctional nanostructures containing 3, 4 or even 5 different functionalities can improve the current state of the art in making nanostructures. On the other hand, it complicates the synthesis of nanomedicines substantially. While it is obvious that such a synthesis technically compromises clinical translation, one should keep in mind that the characterization and validation of such methods of these systems is even more demanding. Scientists tend to draw images or reaction schemes, which do not display side products in order to simplify complexity, but this does not mean that side products are absent. While for small molecules the separation of side products can be often easily performed by chromatographic methods, these approaches are often impossible for polymers, polymer aggregates or colloidal systems. The reason for this fact is simple. Even small differences in molecular structure of small molecules change their properties, for example polarity and molecular mass, substantially, which enables separation. In contrast, side reactions only alter some parts of polymer or nanoparticle and, therefore, usually do not change particle properties in such a way that separation is possible.

In the same line, a $20+$ step organic synthesis as well as the synthesis and functionalization of a generation 4 or 5 dendrimer is an art itself and may yield potent nanomedicines, but massively complicate costeffective industrial production. Related to this point, it has to be kept in mind that the easiest way to actually hinder clinical translation is a synthetic method, which is not scalable to amounts of nanomedicine needed to treat thousands or even millions of patients. Although, amounts differ with respect to the desired application, they can easily reach kilograms or even tons, which have to be produced at the same level of quality.

By contrast, the prejudice remains - at least in polymer science - that the polymeric material or nanoparticles need to be well-defined (narrow size or molecular weight distribution) to enable clinical translation. This statement is already rebutted by the approved drug Copaxone $^{\circledR}$ (Teva Pharmaceutical Industries, Petach Tikva, Israel) [1] and the clinical Phase 1 trial of Crystal therapeutics $\left(\mathrm{CriPec}^{\circledR}\right.$ docetaxel; Cristal Therapeutics, Maastricht, The Netherlands) [12], which are based on copolymers with broad molecular weight distributions. Nevertheless, both materials are safe at therapeutic doses and can be synthesized in a reproducible manner. It can be concluded from this, that more uniform materials may help to correlate material properties to a chemical structure [13,14] but are, by no means, a requirement for the development of nanosized drugs.

Nevertheless, a particle of high internal complexity complicates translation into a applicable drug. Thus, scientists have to critically reflect, if there is a real biological or medical need for all what can be done from a synthetic point of view. Reducing complexity to the minimal biological or medical need seems to be the primary task in nanoparticle synthesis to generate nanosized drugs or imaging agents.

Another prominent example of overgeneralization is the enhanced permeability and retention (EPR) effect, 
which describes an enhanced permeability (leakiness) of tumor (neo)vasculature paired with an inefficient lymphatic drainage in the tumorous tissue [15]. This pathology can lead to an accumulation of nanosized objects $\left(\mathrm{R}_{\mathrm{h}}>5 \mathrm{~nm}\right)$ at the tumor side. But it has to be kept in mind that elongated circulation times (blood half life time $>12 \mathrm{~h}$ ) are required to really take profit of this phenomenon [16]. More importantly, the tumor needs to be well vascularized and the vasculature has to be leaky, which is often not the case. Additionally, leakiness differs between tumor types as well as individual patients [17]. And even in 'EPR-positive' tumors in animal models, maximum accumulation observed peaks at around $10 \%$ of the injected dose and tumor-to-blood drug concentration ratios of 10-30 [18]. Without any doubt, even these effects can have a therapeutic impact in comparison to a standard chemotherapy where often less than $1 \%$ of the drug reaches the tumor [19]. Additionally, a simple reduction of adverse effects due to reduced off-target distribution of the drug can increase the maximum tolerated doses, enabling higher drug doses in patients. This increase in drug dose can result in elevated drug levels at the diseased tissue and thus can yield a more pronounced therapeutic effect as long as the active drug is efficiently released from its carrier [20]. It has been demonstrated by Hiroshi Maeda, Kazunori Kataoka and others that an EPR-based passive delivery approach can cure cancer patients or at least elongate survival substantially [21], but it is by no means a general gateway to solid tumors. Therefore, patients having 'EPR-positive' tumors need to be identified and can then profit from EPR-based therapy, which underlines the importance of imaging or theranostic agents [22].

Related to the passive tumor accumulation by the EPR effect, active targeting seems to be another often misunderstood and maybe even more oversimplified concept. In this approach, a targeting moiety is expected to bind to a cellular antigen, highly expressed on target cells and therefore enhances cellular uptake and achieves cell specificity of the attached nanomedicine. While this approach works often nicely on cells in a petri dish, it often fails in vivo [23]. The reasons for this are for sure again complex, for example, protein corona formation, among others, but at least some points can be considered already at the initial stages of the development of targeted delivery or imaging systems. First of all, it has to be kept in mind that active targeting can only work if the target cell and the corresponding antigen (receptor) are accessible and ideally exclusively expressed at the target site.

Second, to interact with an antigen the ligand has to come in close proximity to it. In the case of cancer the nanomedicine has to reach the tumor cells inside the tumor before any kind of targeted cell uptake can occur. Therefore, the nanomedicine has to reach the tumorous tissue on its own, likely by the EPR effect, which is usually described as passive tumor targeting. In respect to this, a differentiation between active and passive targeting is at least for solid tumor an oversimplification itself, because it implies that both approaches are opposed to each other. But this is simply not the case. Without passive accumulation of the nanoparticle at the tumor tissue active targeting does not even have a chance to take place.

Keeping in mind that lymphatic drainage is already drastically reduced in solid tumors, it appears reasonable that several papers come to the conclusion that receptor targeting does not substantially increase accumulation of nanomedicines in tumors [20]. Furthermore, it is often questionable if active targeting is necessary for the delivery of chemotherapeutic agents. Drugs like doxorubicin, daunorubicin, paclitaxel or docetaxel do not require intracellular delivery. Even as the free drug they can reach their intracellular target. Receptor-mediated uptake, however, may increase intracellular drug levels by overcoming multidrug resistance in certain cancer cells. For other bioactive molecules like proteins, nucleotides or hormones, cell uptake and translocalization is often required to achieve a therapeutic effect, e.g., RNAi. In this respect, active targeting may become a necessity to achieve a therapeutic effect [24].

The idea of functional nanoparticles specifically designed to engage with certain cells in a patient as the 'magic bullet' discourse suggests, is likely to remain a conceptual idea [25]. Although target cells are accessible, like in the case of immune cells, cellular uptake remains a process of probability and thus cannot be fully specific, but active targeting can facilitate uptake, enhance accumulation and may direct intracellular translocalization. After having identified the right target, this enhanced cellular uptake can be already sufficient to achieve therapeutic effects and may lead to novel therapies even in the absence of perfect target specificity.

In conclusion, we know that nanomedicines - even designed to be as simple as required - are at least semicomplex, because such systems have to reproducibly synthesized and characterized. We have learned that drug delivery within the body is complex. And we understand that the biology of many diseases, e.g. cancer, is highly complex. Thus, we cannot get around complexity in the development of nanomedicines. As I am saying in this paper, we have to identify the minimal level of complexity that still leads to a therapeutic effect but also allows for clinical translation. On the other hand, scientist have to carefully evaluate general concepts in the field, because these are always based on 
simplification. What is true for one particular kind of nanomedicine or better one specific system maybe not true for a different one even if size and morphology are identical. Concepts and models can be simple, maybe they need to understand them easily, but therefore we all have to pay the price of losing accuracy. Therefore, scientists need to be aware of the risk that a conclusion based on validated data can become a false statement due to oversimplification or overgeneralization.

In this respect one should end the pursuit of magic bullets, which exclusively tackles a disease in the absence of systemic effects, but try to develop realistic therapeutic concepts, in which doses are minimized, requirements on nanomedicines reduced and effects maximized. Nanoparticle based immune therapies may be such a realistic concept. Hence it is advisable that we need to end the pursuit of complexity with respect to nanoparticle design and therapeutic con-

\section{References}

1 Strebhardt K, Ullrich A. Paul Ehrlich's magical bullet concept: 100 years of progress. Nat. Rev. Cancer 8, 473-480 (2008).

2 Duncan R, Gaspar R. Nanomedicine(s) under the microscope. Mol. Pharm. 8(6), 2101-2141 (2011).

3 Etheridge ML, Campbell SA, Erdman AG, Haynes CL, Wolf SM, McCullough J. The big picture on nanomedicine: the state of investigational and approved nanomedicine products. Nanomedicine 9, 1-14 (2013).

4 Lammers T. SMART drug delivery systems: back to the future vs clinical reality. Int. J. Pharm. 454(1), 527-529 (2013).

5 Grainger DW. Connecting drug delivery reality to smart materials design. Int. J. Pharm. 454(1), 521-524 (2013).

6 Dimitrov DS, Marks JD. Therapeutic antibodies: current state and future trends - is a paradigm change coming soon? Methods Mol. Biol. 525, i-xiii (2009).

7 Allen TM, Cullis PR. Liposomal drug delivery systems: from concept to clinical applications. Adv. Drug Deliv. Rev. 65, 36-48 (2013).

8 Pelegri-O'Day EM, Lin EW, Maynard HD. Therapeutic protein-polymer conjugates: advancing beyond PEGylation. J. Am. Chem. Soc. 136 (41), 14323-14332 (2014).

9 Schwendeman SP, Shah RB, Bailey BA, Schwendeman AS. Injectable controlled release depots for large molecules. J. Control. Release 190, 240-253 (2014).

10 Talelli M, Barz M, Rijcken CJ, Kiessling F, Hennink WE, Lammers T. Core-crosslinked polymeric micelles: Principles, preparation, biomedical applications and clinical translation. Nano Today 10 (1), 93-117 (2015).

11 Aharoni R. Immunomodulation neuroprotection and remyelination - the fundamental therapeutic effects of glatiramer acetate: a critical review. J. Autoimmun. 54, 81-92 (2014).

12 Hu Q, Rijcken CJ, Bansal R, Hennink WE, Storm G, Prakash J. Complete regression of breast tumour with a single cepts in already complex systems. A pursuit of simplicity may finally pave the way to nanosized drugs able to cure or at least improve the quality of life of patients.

\section{Acknowledgements}

The author thanks Professor R Zentel, Professor Dr Michael Schillmeier and the author's group for fruitful discussions during the preparation of this editorial article.

\section{Financial \& competing interests disclosure}

Financial support by the German Research Council (SFB 1066), CINEMA and the NMFZ Mainz is gratefully acknowledged. The author has no other relevant affiliations or financial involvement with any organization or entity with a financial interest in or financial conflict with the subject matter or materials discussed in the manuscript apart from those disclosed.

No writing assistance was utilized in the production of this manuscript.

dose of docetaxel-entrapped core-cross-linked polymeric micelles. Biomaterials 53, 370-378 (2015).

13 Barz M, Luxenhofer R, Zentel R, Vicent MJ. Defined polymers for therapeutic applications: from structureproperty relationships to better defined therapeutics. Polym. Chem. 2, 1900-1918 (2011).

14 Klinker K, Barz M. Polypept(o)ides - hybrid systems based on polypeptides and polypeptoids. Macromol. Rapid Commun. doi: 10.1002/marc.201500403 (2015) (Epub ahead of print).

15 Matsumura Y, Maeda H. A new concept for macromolecular therapeutics in cancer chemotherapy: mechanism of tumoritropic accumulation of proteins and the antitumor agent SMANCS. Cancer Res. 46, 6387-6392 (1986).

16 Torchilin V. Tumor delivery of macromolecular drugs based on the EPR effect. Adv. Drug Deliv. Rev. 63(3), 131-135 (2011).

17 Maeda H, Wu J, Sawa T, Matsumura Y, Hori K. Tumor vascular permeability and the EPR effect in macromolecular therapeutics: a review. J. Control. Release 65(1-2), 271-284 (2000).

18 Maeda H, Bharate GY, Daruwalla J. Polymeric drugs for efficient tumor-targeted drug delivery based on EPR-effect. Eur. J. Pharm. Biopharm. 71, 409-419 (2009).

19 Allen TM, Cullis PR. Drug delivery systems: entering the mainstream. Science 303(5665), 1818-1822 (2004).

20 Lammers T, Hennink WE, Storm G. Tumour-targeted nanomedicines: principles and practice. Br. J. Cancer 99 , 392-397 (2008).

21 Cabral H, Kataoka K. Progress of drug-loaded polymeric micelles into clinical studies. J. Control. Release 190, 465-476 (2014).

22 Lammers T, Aime S, Hennink WE, Storm G, Kiessling F. Theranostic nanomedicine. Acc. Chem. Res. 44(10), 1029-1038 (2011). 
23 Kunjachan S, Pola R, Gremse F et al. Passive versus active tumor targeting using RGD- and NGR-modified polymeric nanomedicines. Nano Lett. 14(2), 972-981 (2014).

24 Vachutinsky Y, Oba M, Miyata K et al. Antiangiogenic gene therapy of experimental pancreatic tumor by sFlt-1 plasmid DNA carried by RGD-modified crosslinked polyplex micelles. J. Control. Release 149(1), 51-57 (2011).

25 Bertrand N, Wu J, Xu X, Kamaly N, Farokhzad O. Cancer nanotechnology: the impact of passive and active targeting in the era of modern cancer biology. Adv. Drug Deliv. Rev. 66, 2-25 (2014). 